\title{
On the Cauchy problem for a class of hyperbolic operators with triple characteristics
}

\author{
Annamaria Barbagallo ${ }^{1}$. Vincenzo Esposito ${ }^{1}$
}

Received: 9 July 2020 / Accepted: 1 October 2020 / Published online: 15 October 2020

(c) The Author(s) 2020

\section{Abstract}

The Cauchy problem for a class of hyperbolic operators with triple characteristics is analyzed. Some a priori estimates in Sobolev spaces with negative indexes are proved. Subsequently, an existence result for the Cauchy problem is obtained.

Keywords Cauchy problem $\cdot$ Hyperbolic equations $\cdot$ Pseudodifferential operators

Mathematics Subject Classification 35B45 $\cdot 35 \mathrm{~S} 05 \cdot 35 \mathrm{~L} 30$

\section{Introduction}

In the past, many authors studied widely hyperbolic operators with double characteristics, both in the case when there is no transition between different types on the set where the principal symbol vanishes of order 2 (see for instance $[5,8]$ for a general survey) and when there is transition (see [1-4]). The operators are called effectively hyperbolic if the propagation cone $C$ is transversal to the manifold of multiple points (see [8]). Moreover, if this occurs and lower order terms satisfy a generic Ivrii-Petkov vanishing condition, we have well posedness in $C^{\infty}$ (see [7]).

The aim of the paper is to analyze the following class of operators with triple characteristics

$$
\left.P\left(x_{0}, D\right)=D_{x_{0}}^{3}-\left(D_{x_{1}}^{2}+x_{1}^{2} D_{x_{2}}^{2}\right) D_{x_{0}}-b x_{1}^{3} D_{x_{2}}^{3}, \quad \text { in } \Omega=\right] 0,+\infty\left[\times \mathbb{R}^{2},\right.
$$

where $D_{x_{j}}=\frac{1}{i} \partial_{x_{j}}, j=0,1,2$, under hyperbolicity assumptions, namely $|b| \leq \frac{2}{3}$. Such a class of operators has been considered in [6], for example operators whose propagation cone is not transversal to the triple characteristic manifold. The authors

Annamaria Barbagallo

annamaria.barbagallo@unina.it

1 Department of Mathematics and Applications "R. Caccioppoli”, University of Naples Federico II, Via Cinthia - Monte S. Angelo, 80126 Naples, Italy 
prove a well posedness result in the Gevrey category for a simple hyperbolic operator with triple characteristics and whose propagation cone is not transversal to the triple manifold. Furthermore they estimate the precise Gevrey threshold, by exhibiting a special class of solutions, through which we can violate weak necessary solvability conditions. More precisely, let $x=\left(x_{0}, x^{\prime}\right)$ where $x^{\prime}=\left(x_{1}, x_{2}\right)$, let $\xi=\left(\xi_{0}, \xi^{\prime}\right)$, where $\xi^{\prime}=\left(\xi_{1}, \xi_{2}\right)$. In [6], the authors study the well posedness of the following Cauchy problem

$$
\left\{\begin{array}{l}
P u=0, \quad \text { in } \Omega=] 0,+\infty\left[\times \mathbb{R}^{2},\right. \\
D_{x_{j}} u\left(0, x^{\prime}\right)=\phi_{j}\left(x^{\prime}\right), \quad j=0,1,2
\end{array}\right.
$$

with $\phi_{j}\left(x^{\prime}\right) \in \gamma^{(s)}\left(\mathbb{R}^{2}\right), j=0,1,2$, where $\gamma^{(s)}\left(\mathbb{R}^{2}\right)$ is the Gevrey $s$ class. They obtained that the Cauchy problem for $P$ is well posed in the Gevrey 2 class assuming that $b^{2}<\frac{4}{27}$. Moreover, if $s>2$, it is possible to choose $\left.b \in\right] 0, \frac{2}{3 \sqrt{3}}[$ such that the Cauchy problem for $P$ is not locally solvable at the origin in the Gevrey $s$ class.

In this paper, instead, we investigate on the well posedness of the Cauchy problem

$$
\left\{\begin{array}{l}
P u=f, \quad \text { in } \Omega, \\
D_{x_{j}} u\left(0, x^{\prime}\right)=0, j=0,1,2,
\end{array}\right.
$$

with $f \in H^{r}(\Omega)$, in the Sobolev spaces, obtaining an existence result for solutions.

Let us set

$$
Q=-\partial_{x_{0}}^{3}+\left(\partial_{x_{1}}^{2}+x_{1}^{2} \partial_{x_{2}}^{2}\right) \partial_{x_{0}}+b x_{1}^{3} \partial_{x_{2}}^{3}, \quad \text { in } \Omega
$$

It results

$$
P u=i Q u, \quad \text { in } \Omega \text {. }
$$

As a consequence, problem (1) becomes

$$
\left\{\begin{array}{l}
Q u=g, \quad \text { in } \Omega, \\
\partial_{x_{j}} u\left(0, x^{\prime}\right)=0, j=0,1,2,
\end{array}\right.
$$

where we set $g=i f$, in $\Omega$, with $g$ real function. The main result of the paper is the following.

Theorem 1 Let $f \in H_{l o c}^{r}(\bar{\Omega})$, with $r \geq 5$. For every $h, T>0$, the Cauchy problem (1) admits a solution $u \in H^{r-2}\left(\Omega_{h, T}\right)$, where $\Omega_{h, T}=\left[0, h[\times]-T, T\left[^{2}\right.\right.$.

The rest of the paper is organized as follows. Section 2 deals with some preliminary notations and definitions. In Sect. 3 some a priori estimates are established. Section 4 is devoted to obtain a priori estimates in Sobolev spaces with negative indexes. Finally, the existence result for solutions to the Cauchy problem are proved in Sect. 5. 


\section{Notations and preliminaries}

Let $\alpha=\left(\alpha_{0}, \alpha_{1}, \alpha_{2}\right) \in \mathbb{N}_{0}^{3}$. Let $\partial^{\alpha}$ be the derivative of order $|\alpha|$, let $\partial_{x_{j}}^{h}$ be the derivative of order $h$ with respect to $x_{j}$ and let $\partial_{x_{j}, x_{p}}^{h}$ be the derivative of order $h$ with respect to $x_{j}$ and $x_{p}$.

We indicate the $L^{2}$-scalar product, the $L^{2}$-norm and the $H^{r}$-norm $\left(r \in \mathbb{N}_{0}\right)$ by $(\cdot, \cdot)$, $\|\cdot\|$ and $\|\cdot\|_{H^{r}}$ respectively.

Let $\Omega$ be an open subset of $\mathbb{R}^{3}$. Let $C_{0}^{\infty}(\bar{\Omega})$ be the space of the restrictions to $\bar{\Omega}$ of functions belonging to $C_{0}^{\infty}\left(\mathbb{R}^{3}\right)$. For each $K \subseteq \bar{\Omega}$ compact set, let $C_{0}^{\infty}(K)$ be the set of functions $\varphi \in C_{0}^{\infty}(\bar{\Omega})$ having support contained in $K$. Let $S\left(\mathbb{R}^{3}\right)$ be the space of rapidly decreasing functions. In particular, let $S(\bar{\Omega})$ be the space of the restrictions to $\bar{\Omega}$ of functions belonging to $S\left(\mathbb{R}^{3}\right)$.

Let $\Omega=\left[0,+\infty[\times] a_{1}, b_{1}[\times]-\infty,+\infty[\right.$ and let $s \in \mathbb{R}$, let us denote by $\| \cdot$ $\|_{H^{0,0, s}(\bar{\Omega})}$ the norm given by

$$
\begin{aligned}
\|u\|_{H^{0,0, s}(\bar{\Omega})}^{2}= & \int_{0}^{+\infty} d x_{0} \int_{a_{1}}^{b_{1}} d x_{1} \\
& \int_{-\infty}^{+\infty} \frac{1}{2 \pi}\left(1+\left|\xi_{2}\right|^{2}\right)^{s}\left|\widehat{u}\left(x_{0}, x_{1}, \xi_{2}\right)\right|^{2} d \xi_{2}, \quad \forall u \in C_{0}^{\infty}(\bar{\Omega}),
\end{aligned}
$$

where the Fourier transform is performed only with respect to the variable $x_{2}$. Moreover, let us denote by $A_{s}$ the pseudodifferential operator given by

$$
A_{s} u(x)=\int_{-\infty}^{+\infty} \frac{1}{2 \pi} e^{i x_{2} \cdot \xi_{2}}\left(1+\left|\xi_{2}\right|^{2}\right)^{\frac{s}{2}} \widehat{u}\left(x_{0}, x_{1}, \xi_{2}\right) d \xi_{2}, \quad \forall u \in C_{0}^{\infty}(\bar{\Omega})
$$

Let us recall that $A_{s}: C_{0}^{\infty}(\bar{\Omega}) \rightarrow C^{\infty}(\bar{\Omega})$. For every $\varphi\left(x_{2}\right) \in C_{0}^{\infty}(\mathbb{R})$, the operator $\varphi A_{s} u$ extends to a linear continuous operator from $H_{c o m p}^{0,0, r} .(\bar{\Omega})$ to $H_{l o c}^{0,0, r-s}(\bar{\Omega})$, where $r, s \in \mathbb{R}$. In particular, in $\Omega_{k}=\left[0, k[\times] a_{1}, b_{1}[\times]-\infty,+\infty[\right.$, for $k>0$, we denote by $H^{0,0, s}\left(\Omega_{k}\right)$ the space of all $u \in H^{0,0, s}(\Omega)$ such that supp $u \subseteq \Omega_{k}$. Moreover, denoted by $\mathcal{U}_{x_{2}}$ the projection of supp $u$ on the axis $x_{2}$, if supp $\varphi \subseteq \mathbb{R} \backslash \mathcal{U}_{x_{2}}$, then $\varphi A_{s} u$ is regularizing with respect to the variable $x_{2}$, namely it results:

$$
\left\|\varphi A_{s} u\right\|_{H^{0,0, r}} \leq c\|u\|_{H^{0,0, r^{\prime}}}, \quad \forall r, r^{\prime} \in \mathbb{R}, u \in C^{\infty}(\bar{\Omega}) .
$$

The norms $\|u\|_{H^{0,0, s}(\Omega)}$ and $\left\|A_{s} u\right\|_{L^{2}(\Omega)}$ are equivalent for any $s \in \mathbb{R}$.

Let $s \in \mathbb{R}$ and $p \geq 0$. Let $H^{p, s}\left(\mathbb{R}^{3}\right)$ be the space of distributions $U$ into $\mathbb{R}^{3}$ such that

$$
\|u\|_{H^{p, s}\left(\mathbb{R}^{3}\right)}^{2}=\frac{1}{2 \pi} \sum_{|h| \leq p} \int_{\mathbb{R}^{3}}\left(1+\left|\xi_{2}\right|^{2}\right)^{s}\left|\partial_{x_{0}, x_{1}}^{h} \widehat{U}\left(x_{0}, x_{1}, \xi_{2}\right)\right|^{2} d x_{0} d x_{1} d \xi_{2}<+\infty .
$$


At last, let $H^{p, s}(\Omega)$ be the space of the restrictions to $\Omega$ of elements of $H^{p, s}\left(\mathbb{R}^{3}\right)$ endowed with the norm

$$
\|u\|_{H^{p, s}(\Omega)}=\inf _{\substack{\left.U \in H^{p, s}\left(\mathbb{R}^{3}\right) \\ U\right|_{\Omega}=u}}\|U\|_{H^{p, s}\left(\mathbb{R}^{3}\right)} .
$$

\section{A priori estimates}

The following preliminary result holds (see [1], Lemma 3.1).

Lemma 1 Let $u \in S(\bar{\Omega})$ and let $p, \alpha_{0}, \alpha_{1}, \alpha_{2} \in \mathbb{N}_{0}$. Then

$$
\left\|x_{0}^{\frac{p}{2}} \partial^{\alpha_{0}, \alpha_{1}, \alpha_{2}} u\right\| \leq \frac{2}{p+1}\left\|x_{0}^{\frac{p+2}{2}} \partial^{\alpha_{0}+1, \alpha_{1}, \alpha_{2}} u\right\| .
$$

Now, we establish a useful estimate.

Lemma 2 Let $u \in C_{0}^{\infty}\left(\left[0,+\infty\left[\times \mathbb{R}^{2}\right)\right.\right.$ such that $\operatorname{supp} u \subseteq\left[0, h[\times]-T, T\left[^{2}\right.\right.$. Let $\varphi \in C_{0}^{\infty}(\mathbb{R})$ such that $\left.\operatorname{supp} \varphi \subseteq \mathbb{R} \backslash\right]-n T, n T[$, with $n \geq 2$. For every $r \leq 0, s \in \mathbb{R}$ and $p \geq s+r$, it results

$$
\left\|\varphi A_{s} u\right\|_{L^{2}(\Omega)} \leq \frac{c_{p, r, s}}{[(n-1) T]^{p}}\|u\|_{H^{0,0, r}(\Omega)} .
$$

Proof In order to obtain the claim, we follow analogous techniques used in the proof of Lemma 3.2 in [3]. For the reader's convenience, we present the demonstration. We have

$$
\begin{aligned}
\left(\varphi A_{s} u\right)(x)= & \frac{1}{2 \pi} \int_{-\infty}^{+\infty} e^{i x_{2} \xi_{2}} \varphi\left(x_{2}\right)\left(1+\left|\xi_{2}\right|^{2}\right)^{\frac{s}{2}} \widehat{u}\left(x_{0}, x_{1}, \xi_{2}\right) d \xi_{2} \\
= & \frac{1}{2 \pi} \iint_{\mathbb{R}^{2}} e^{i\left(x_{2}-y_{2}\right) \xi_{2}} \varphi\left(x_{2}\right)\left(1+\left|\xi_{2}\right|^{2}\right)^{\frac{s}{2}} u\left(x_{0}, x_{1}, y_{2}\right) d y_{2} d \xi_{2} \\
= & \frac{i^{m}}{2 \pi} \iint_{\mathbb{R}^{2}} e^{i\left(x_{2}-y_{2}\right) \xi_{2}} \frac{\varphi\left(x_{2}\right) u\left(x_{0}, x_{1}, y_{2}\right)}{\left(x_{2}-y_{2}\right)^{m}} \partial_{\xi_{2}}^{m}\left(1+\left|\xi_{2}\right|^{2}\right)^{\frac{s}{2}} d y_{2} d \xi_{2} \\
= & \frac{i^{m} \varphi\left(x_{2}\right)}{2 \pi} \int_{-\infty}^{+\infty} \partial_{\xi_{2}}^{m}\left(1+\left|\xi_{2}\right|^{2}\right)^{\frac{s}{2}} d \xi_{2} \int_{-\infty}^{+\infty} e^{i\left(x_{2}-y_{2}\right) \xi_{2}} \\
& u\left(x_{0}, x_{1}, y_{2}\right) \frac{\psi\left(\frac{x_{2}-y_{2}}{(n-1) T}\right)}{\left(x_{2}-y_{2}\right)^{m}} d y_{2},
\end{aligned}
$$

where $m \in \mathbb{N}$ and $\psi \in C^{\infty}(\mathbb{R})$ such that $\psi(\tau)=1$ if $|\tau| \geq 1, \psi(\tau)=0$ if $|\tau| \leq \frac{1}{2}$. 
By using (5), we get

$$
\begin{gathered}
\left(\varphi A_{s} u\right)(x)=\frac{i^{m} \varphi\left(x_{2}\right)}{2 \pi} \int_{-\infty}^{+\infty} \partial_{\xi_{2}}^{m}\left(1+\left|\xi_{2}\right|^{2}\right)^{\frac{s}{2}} u\left(x_{0}, x_{1}, x_{2}\right) \\
*\left(\psi\left(\frac{x_{2}}{(n-1) T}\right) \frac{e^{i x_{2} \xi_{2}}}{x_{2}^{m}}\right) d \xi_{2}
\end{gathered}
$$

and also

$$
\begin{aligned}
\mathcal{F}_{x_{2}}\left(\varphi A_{s} u\right)\left(x_{0}, x_{1}, \eta_{2}\right)= & \frac{i^{m} \widehat{\varphi}\left(\eta_{2}\right)}{2 \pi} * \int_{-\infty}^{+\infty} \partial_{\xi_{2}}^{m}\left(1+\left|\xi_{2}\right|^{2}\right)^{\frac{s}{2}} \widehat{u}\left(x_{0}, x_{1}, \eta_{2}\right) \\
& \cdot \mathcal{F}_{x_{2}}\left(\psi\left(\frac{x_{2}}{(n-1) T}\right) \frac{e^{i x_{2} \xi_{2}}}{x_{2}^{m}}\right) d \xi_{2},
\end{aligned}
$$

where

$$
\mathcal{F}_{x_{2}}\left(\psi\left(\frac{x_{2}}{(n-1) T}\right) \frac{e^{i x_{2} \xi_{2}}}{x_{2}^{m}}\right)=\int_{-\infty}^{+\infty} e^{i x_{2}\left(\xi_{2}-\eta_{2}\right)} \psi\left(\frac{x_{2}}{(n-1) T}\right) \frac{1}{x_{2}^{m}} d x_{2},
$$

Easily, we deduce

$$
\begin{aligned}
(1 & \left.+\left(\xi_{2}-\eta_{2}\right)^{r}\right) \mathcal{F}_{x_{2}}\left(\psi\left(\frac{x_{2}}{(n-1) T}\right) \frac{e^{i x_{2} \xi_{2}}}{x_{2}^{m}}\right) \\
& =\int_{-\infty}^{+\infty} e^{i x_{2}\left(\xi_{2}-\eta_{2}\right)} \psi\left(\frac{x_{2}}{(n-1) T}\right) \frac{1}{x_{2}^{m}} d x_{2} \\
& +i^{r} \sum_{j=0}^{r}\left(\begin{array}{l}
r \\
j
\end{array}\right) \int_{-\infty}^{+\infty} e^{i x_{2}\left(\xi_{2}-\eta_{2}\right)} \partial_{x_{2}}^{j} \psi\left(\frac{x_{2}}{(n-1) T}\right) \partial_{x_{2}}^{r-j} \frac{1}{x_{2}^{m}} d x_{2},
\end{aligned}
$$

and, then,

$$
\left|\mathcal{F}_{x_{2}}\left(\psi\left(\frac{x_{2}}{(n-1) T}\right) \frac{e^{i x_{2} \xi_{2}}}{x_{2}^{m}}\right)\right| \leq \frac{c_{r, m}}{\left(1+\left(\xi_{2}-\eta_{2}\right)^{2}\right)^{\frac{r}{2}}}\left(\frac{1}{(n-1) T}\right)^{m-2} .
$$

Making use of (6) and (7), we obtain

$$
\begin{aligned}
\left\|\varphi A_{s} u\right\|= & \left\|\mathcal{F}_{x_{2}}\left(\varphi A_{s} u\right)\right\| \\
\leq & \frac{1}{2 \pi}\|\widehat{\varphi}\|_{L^{1}(\mathbb{R})} \| \int_{-\infty}^{+\infty} \partial_{\xi_{2}}^{m}\left(1+\left|\xi_{2}\right|^{2}\right)^{\frac{s}{2}} \widehat{u}\left(x_{0}, x_{1}, \eta_{2}\right) \\
& \mathcal{F}_{x_{2}}\left(\psi\left(\frac{x_{2}}{(n-1) T}\right) \frac{e^{i x_{2} \xi_{2}}}{x_{2}^{m}}\right) d \xi_{2} \|_{L^{2}(\Omega)} \\
\leq & c \int_{-\infty}^{+\infty} \| \partial_{\xi_{2}}^{m}\left(1+\left|\xi_{2}\right|^{2}\right)^{\frac{s}{2}} \widehat{u}\left(x_{0}, x_{1}, \eta_{2}\right)
\end{aligned}
$$




$$
\begin{gathered}
\mathcal{F}_{x_{2}}\left(\psi\left(\frac{x_{2}}{(n-1) T}\right) \frac{e^{i x_{2} \xi_{2}}}{x_{2}^{m}}\right) \|_{L^{2}(\Omega)} d \xi_{2} \\
\leq \frac{c_{r, m}}{[(n-1) T]^{m-2}} \int_{-\infty}^{+\infty} \frac{\left\|\partial_{\xi_{2}}^{m}\left(1+\left|\xi_{2}\right|^{2}\right)^{\frac{s}{2}} \widehat{u}\left(x_{0}, x_{1}, \eta_{2}\right)\right\|_{L^{2}(\Omega)}}{\left(1+\left(\xi_{2}-\eta_{2}\right)^{2}\right)^{\frac{r}{2}}} d \xi_{2} .
\end{gathered}
$$

From the previous inequality and the Peetre inequality (see [9], pag. 17), it follows

$$
\begin{aligned}
& \left\|\varphi A_{s} u\right\|_{L^{2}(\Omega)} \leq \frac{c_{r, m, s}}{[(n-1) T]^{m-2}} \\
& \int_{-\infty}^{+\infty} \frac{\left\|\left(1+\left|\xi_{2}\right|^{2}\right)^{\frac{s}{2}-\frac{m+1}{2}} \widehat{u}\left(x_{0}, x_{1}, \eta_{2}\right)\right\|_{L^{2}(\Omega)}}{\left(1+\left(\xi_{2}-\eta_{2}\right)^{2}\right)^{\frac{r}{2}}} d \xi_{2} .
\end{aligned}
$$

If $m \geq s+r+2$, setting $p=m-2$ in (8), it results

$$
\left\|\varphi A_{s} u\right\|_{L^{2}(\Omega)} \leq \frac{c_{p, r, s}}{[(n-1) T]^{p}}\|u\|_{H^{0,0, r}(\Omega)},
$$

where $c_{p, r, s}$ is independent of $n$ and $T$.

Taking into account Lemma 2, we deduce

Lemma 3 Let $\varphi \in C_{0}^{\infty}(\mathbb{R})$ such that $\varphi(\tau)=0$, for $|\tau| \leq 1$. For every $\varepsilon>0$, for every $r \leq 0$ and $s \in \mathbb{R}$ there exists $n>1$ such that

$$
\left\|\varphi\left(\frac{x_{2}}{(n-1) T}\right) A_{s} u\right\|_{L^{2}(\Omega)} \leq \varepsilon\|u\|_{H^{0,0, r}(\Omega)} .
$$

In the following, we establish a priori estimates in $L^{2}\left(\Omega_{T}\right)$, where $\Omega_{h, T}=$ $\left[0, h[\times]-T, T\left[^{2}\right.\right.$, for functions belonging to $C_{0}^{\infty}\left(\Omega_{h, T}\right)$.

Theorem 2 For every $h, T>0$, there exists a positive constant $c$ such that

$$
\left\|\partial_{x_{0}} u\right\|+\|u\| \leq c\left(\left\|\partial_{x_{1}} Q u\right\|+\left\|\partial_{x_{2}} Q u\right\|\right), \quad \forall u \in C_{0}^{\infty}(\bar{\Omega}): \operatorname{supp} u \subseteq \Omega_{h, T}
$$

Proof By means of a translation with respect to $x_{2}$ in $T$, we consider the function

$$
\left.v\left(x_{0}, x_{1}, x_{2}\right)=u\left(x_{0}, x_{1}, x_{2}-T\right), \quad \text { in } \Omega_{T}^{\prime}=\right] 0,+\infty[\times]-T, T[\times] 0,2 T[.
$$

We extend the function $v$ in even manner in $]-2 T, 2 T[$. It results

$$
\left.v\left(x_{0}, x_{1},-x_{2}\right)=v\left(x_{0}, x_{1}, x_{2}\right), \quad \text { in } \Omega_{T}^{\prime \prime}=\right] 0,+\infty[\times]-T, T[\times]-2 T, 2 T[.
$$


We consider the following Fourier development of the function $v$ :

$$
\begin{aligned}
v\left(x_{0}, x_{1}, x_{2}\right) & =\sum_{n=-\infty}^{+\infty} c_{n}\left(x_{0}, x_{1}\right) \frac{e^{i n \omega_{0} x_{2}}}{2 \sqrt{T}} \\
& =\sum_{n=-\infty}^{+\infty} v_{n}\left(x_{0}, x_{1}, x_{2}\right)
\end{aligned}
$$

where $\omega_{0}=\frac{2 \pi}{4 T}=\frac{\pi}{2 T}$ and

$$
c_{n}\left(x_{0}, x_{1}\right)=\frac{1}{2 \sqrt{T}} \int_{-2 T}^{2 T} v\left(x_{0}, x_{1}, x_{2}\right) e^{-i n \omega_{0} x_{2}} d x_{2}
$$

We remark that the Fourier coefficients $c_{n}$ are real. We apply the operator $Q$ to $v_{n}$ obtaining

$$
\begin{aligned}
Q v_{n}\left(x_{0}, x_{1}, x_{2}\right)= & \frac{e^{i n \omega_{0} x_{2}}}{2 \sqrt{T}}\left[-\partial_{x_{0}}^{3} c_{n}\left(x_{0}, x_{1}\right)+\partial_{x_{1}}^{2} \partial_{x_{0}} c_{n}\left(x_{0}, x_{1}\right)\right. \\
& \left.-n^{2} \omega_{0}^{2} x_{1}^{2} \partial_{x_{0}} c_{n}\left(x_{0}, x_{1}\right)-i n^{3} \omega_{0}^{3} b x_{1}^{3} c_{n}\left(x_{0}, x_{1}\right)\right] \\
= & L_{n} c_{n}\left(x_{0}, x_{1}\right) \frac{e^{i n \omega_{0} x_{2}}}{2 \sqrt{T}},
\end{aligned}
$$

where we set

$$
\begin{aligned}
L_{n} c_{n}\left(x_{0}, x_{1}\right)= & -\partial_{x_{0}}^{3} c_{n}\left(x_{0}, x_{1}\right)+\partial_{x_{1}}^{2} \partial_{x_{0}} c_{n}\left(x_{0}, x_{1}\right)-n^{2} \omega_{0}^{2} x_{1}^{2} \partial_{x_{0}} c_{n}\left(x_{0}, x_{1}\right) \\
& -i n^{3} \omega_{0}^{3} b x_{1}^{3} c_{n}\left(x_{0}, x_{1}\right)
\end{aligned}
$$

It results

$$
Q v\left(x_{0}, x_{1}, x_{2}\right)=\sum_{n=-\infty}^{+\infty} L_{n} c_{n}\left(x_{0}, x_{1}\right) \frac{e^{i n \omega_{0} x_{2}}}{2 \sqrt{T}}
$$

We estimate the Fourier coefficients $c_{n}\left(x_{0}, x_{1}\right)$ by means of $L_{n} c_{n}\left(x_{0}, x_{1}\right)$ in $L^{2}$. To this aim, let us consider the inner products

$$
\begin{aligned}
& \left(L_{n} c_{n}, x_{0} \partial_{x_{0}}^{2} c_{n}\right)+\left(x_{0} \partial_{x_{0}}^{2} c_{n}, L_{n} c_{n}\right) \\
& =-2\left(\partial_{x_{0}}^{3} c_{n}, x_{0} \partial_{x_{0}}^{2} c_{n}\right)+2\left(\partial_{x_{0}} \partial_{x_{1}}^{2} c_{n}, x_{0} \partial_{x_{0}}^{2} c_{n}\right)-2 n^{2} \omega_{0}^{2}\left(x_{1}^{2} \partial_{x_{0}} c_{n}, x_{0} \partial_{x_{0}}^{2} c_{n}\right) \\
& =2\left\|\partial_{x_{0}}^{2} c_{n}\right\|^{2}-2\left(\partial_{x_{1}} \partial_{x_{0}} c_{n}, x_{0} \partial_{x_{0}}^{2} \partial_{x_{1}} c_{n}\right)+2 n^{2} \omega_{0}^{2}\left\|x_{1} \partial_{x_{0}} c_{n}\right\|^{2} \\
& =2\left\|\partial_{x_{0}}^{2} c_{n}\right\|^{2}+2\left\|\partial_{x_{1}} \partial_{x_{0}} c_{n}\right\|^{2}+2 n^{2} \omega_{0}^{2}\left\|x_{1} \partial_{x_{0}} c_{n}\right\|^{2} .
\end{aligned}
$$


From which we have

$$
\left\|\partial_{x_{0}}^{2} c_{n}\right\|^{2}+\left\|\partial_{x_{1}} \partial_{x_{0}} c_{n}\right\|^{2}+n^{2} \omega_{0}^{2}\left\|x_{1} \partial_{x_{0}} c_{n}\right\|^{2} \leq c\left\|x_{0} L_{n} c_{n}\right\|^{2}
$$

Let us evaluate the inner products

$$
\left(L_{n} \partial_{x_{1}} c_{n}, x_{0} \partial_{x_{0}}^{2} \partial_{x_{1}} c_{n}\right)+\left(x_{0} \partial_{x_{0}}^{2} \partial_{x_{1}} c_{n}, L_{n} \partial_{x_{1}} c_{n}\right)
$$

Proceeding as in (10), we obtain

$$
\begin{aligned}
& \left\|\partial_{x_{0}}^{2} \partial_{x_{1}} c_{n}\right\|^{2}+\left\|\partial_{x_{1}}^{2} \partial_{x_{0}} c_{n}\right\|^{2}+n^{2} \omega_{0}^{2}\left\|x_{1} \partial_{x_{0}} \partial_{x_{1}} c_{n}\right\|^{2} \\
& \quad=\left(L_{n} \partial_{x_{1}} c_{n}, x_{0} \partial_{x_{0}}^{2} \partial_{x_{1}} c_{n}\right)+\left(x_{0} \partial_{x_{0}}^{2} \partial_{x_{1}} c_{n}, L_{n} \partial_{x_{1}} c_{n}\right) \\
& \quad=\left(\partial_{x_{1}} L_{n} c_{n}, x_{0} \partial_{x_{0}}^{2} \partial_{x_{1}} c_{n}\right)+\left(x_{0} \partial_{x_{0}}^{2} \partial_{x_{1}} c_{n}, \partial_{x_{1}} L_{n} c_{n}\right) \\
& \quad+4\left(x_{1} n^{2} \omega_{0}^{2} \partial_{x_{0}} c_{n}, x_{0} \partial_{x_{0}}^{2} \partial_{x_{1}} c_{n}\right)
\end{aligned}
$$

Hence, we deduce

$$
\begin{aligned}
& \frac{1}{n^{2} \omega_{0}^{2}}\left\|\partial_{x_{0}}^{2} \partial_{x_{1}} c_{n}\right\|^{2}+\frac{1}{n^{2} \omega_{0}^{2}}\left\|\partial_{x_{1}}^{2} \partial_{x_{0}} c_{n}\right\|^{2}+\left\|x_{1} \partial_{x_{0}} \partial_{x_{1}} c_{n}\right\|^{2} \\
& \quad \leq \frac{2}{n^{2} \omega_{0}^{2}}\left\|x_{0} \partial_{x_{1}} L_{n} c_{n}\right\|\left\|\partial_{x_{0}}^{2} \partial_{x_{1}} c_{n}\right\|+4\left\|x_{0} x_{1} \partial_{x_{0}} c_{n}\right\|\left\|\partial_{x_{0}}^{2} \partial_{x_{1}} c_{n}\right\|
\end{aligned}
$$

As a consequence, we have

$$
\begin{aligned}
& \frac{1}{n^{2} \omega_{0}^{2}}\left\|\partial_{x_{0}}^{2} \partial_{x_{1}} c_{n}\right\|^{2}+\frac{1}{n^{2} \omega_{0}^{2}}\left\|\partial_{x_{1}}^{2} \partial_{x_{0}} c_{n}\right\|^{2}+\left\|x_{1} \partial_{x_{0}} \partial_{x_{1}} c_{n}\right\|^{2} \\
& \quad \leq \frac{c}{n^{2} \omega_{0}^{2}}\left\|x_{0} \partial_{x_{1}} L_{n} c_{n}\right\|^{2}+c n^{2} \omega_{0}^{2}\left\|x_{1} \partial_{x_{0}} c_{n}\right\|^{2}
\end{aligned}
$$

Making use of (11) and (12), we get

$$
\begin{aligned}
& \left\|\partial_{x_{0}}^{2} c_{n}\right\|^{2}+\left\|\partial_{x_{1}} \partial_{x_{0}} c_{n}\right\|^{2}+n^{2} \omega_{0}^{2}\left\|x_{1} \partial_{x_{0}} c_{n}\right\|^{2} \\
& +\frac{1}{n^{2} \omega_{0}^{2}}\left\|\partial_{x_{0}}^{2} \partial_{x_{1}} c_{n}\right\|^{2}+\frac{1}{n^{2} \omega_{0}^{2}}\left\|\partial_{x_{1}}^{2} \partial_{x_{0}} c_{n}\right\|^{2} \\
& +\left\|x_{1} \partial_{x_{0}} \partial_{x_{1}} c_{n}\right\|^{2} \leq c\left\|x_{0} L_{n} c_{n}\right\|^{2}+\frac{c}{n^{2} \omega_{0}^{2}}\left\|x_{0} \partial_{x_{1}} L_{n} c_{n}\right\|^{2} \\
& \quad=\frac{c}{n^{2} \omega_{0}^{2}}\left\|x_{0} \partial_{x_{1}} L_{n} c_{n}\right\|^{2}+\frac{c}{n^{2} \omega_{0}^{2}}\left\|i x_{0} n \omega_{0} L_{n} c_{n}\right\|^{2} .
\end{aligned}
$$

Let us consider $v \in C_{0}^{\infty}(] 0,+\infty[\times]-T, T[\times] 0,2 T[)$ and we still denote by $v$ its even extension in $] 0,+\infty[\times]-T, T[\times]-2 T, 2 T[$. Let us develop $v$ in Fourier's series with respect to $x_{2}$ : 


$$
v\left(x_{0}, x_{1}, x_{2}\right)=\sum_{n=-\infty}^{+\infty} c_{n}\left(x_{0}, x_{1}\right) \frac{e^{i n \omega_{0} x_{2}}}{2 \sqrt{T}}
$$

from which it follows

$$
Q v\left(x_{0}, x_{1}, x_{2}\right)=\sum_{n=-\infty}^{+\infty} L_{n} c_{n}\left(x_{0}, x_{1}\right) \frac{e^{i n \omega_{0} x_{2}}}{2 \sqrt{T}},
$$

Hence, it results

$$
x_{1} \partial_{x_{0}} \partial_{x_{1}} v\left(x_{0}, x_{1}, x_{2}\right)=\sum_{n=-\infty}^{+\infty} x_{1} \partial_{x_{0}} \partial_{x_{1}} c_{n}\left(x_{0}, x_{1}\right) \frac{e^{i n \omega_{0} x_{2}}}{2 \sqrt{T}} .
$$

Applying the Parseval inequality, we have

$$
\begin{aligned}
\left\|x_{1} \partial_{x_{0}} \partial_{x_{1}} v\right\|^{2} & =\|\| x_{1} \partial_{x_{0}} \partial_{x_{1}} v\left\|_{]-2 T, 2 T[}^{2}\right\|_{] 0,+\infty[\times]-T, T[}^{2} \\
& =\left\|\sum_{n=-\infty}^{+\infty}\left|x_{1} \partial_{x_{0}} \partial_{x_{1}} c_{n}\right|^{2}\right\|_{] 0,+\infty[\times]-T, T[}^{2} \\
& \leq \sum_{n=-\infty}^{+\infty}\left\|x_{1} \partial_{x_{0}} \partial_{x_{1}} c_{n}\right\|^{2} .
\end{aligned}
$$

Taking into account (13) and (14), we obtain

$$
\begin{aligned}
\left\|x_{1} \partial_{x_{0}} \partial_{x_{1}} v\right\|^{2} & \leq \sum_{n=-\infty}^{+\infty}\left\|x_{1} \partial_{x_{0}} \partial_{x_{1}} c_{n}\right\|^{2} \\
& \leq \sum_{n=-\infty}^{+\infty}\left[\frac{c}{n^{2} \omega_{0}^{2}}\left\|x_{0} \partial_{x_{1}} L_{n} c_{n}\right\|^{2}+\frac{c}{n^{2} \omega_{0}^{2}}\left\|i x_{0} n \omega_{0} L_{n} c_{n}\right\|^{2}\right] \\
& \leq \frac{c}{\omega_{0}^{2}} \sum_{n=-\infty}^{+\infty} \frac{1}{n^{2}}\left[\left\|x_{0} \partial_{x_{1}} L_{n} c_{n}\right\|^{2}+\left\|i n x_{0} \omega_{0} L_{n} c_{n}\right\|^{2}\right] .
\end{aligned}
$$

We remark that

$$
x_{0} \partial_{x_{1}} Q v\left(x_{0}, x_{1}, x_{2}\right)=\sum_{n=-\infty}^{+\infty} x_{0} \partial_{x_{1}} L_{n} c_{n}\left(x_{0}, x_{1}\right) \frac{e^{i n \omega_{0} x_{2}}}{2 \sqrt{T}} .
$$

For the Parseval inequality, it results

$$
\begin{aligned}
\left\|x_{0} \partial_{x_{1}} Q v\right\|^{2} & =\|\| x_{0} \partial_{x_{1}} Q v\left\|_{]-2 T, 2 T[}^{2}\right\|_{] 0,+\infty[\times]-T, T[}^{2} \\
& =\left\|\sum_{n=-\infty}^{+\infty}\left|x_{0} \partial_{x_{1}} L_{n} c_{n}\right|^{2}\right\|_{] 0,+\infty[\times]-T, T[}^{2}
\end{aligned}
$$


Moreover, we remark that

$$
x_{0} \partial_{x_{2}} Q v\left(x_{0}, x_{1}, x_{2}\right)=\sum_{n=-\infty}^{+\infty} i n \omega_{0} x_{0} L_{n} c_{n}\left(x_{0}, x_{1}\right) \frac{e^{i n \omega_{0} x_{2}}}{2 \sqrt{T}} .
$$

Applying, again, the Parseval inequality, we have

$$
\begin{aligned}
\left\|x_{0} \partial_{x_{2}} Q v\right\|^{2} & =\|\| x_{0} \partial_{x_{2}} Q v\left\|_{]-2 T, 2 T[}^{2}\right\|_{] 0,+\infty[\times]-T, T[}^{2} \\
& =\left\|\sum_{n=-\infty}^{+\infty}\left|i n \omega_{0} x_{0} L_{n} c_{n}\right|^{2}\right\|_{] 0,+\infty[\times]-T, T[}^{2}
\end{aligned}
$$

Making use of (15), (16) and (17), we obtain

$$
\begin{aligned}
\left\|x_{1} \partial_{x_{0}} \partial_{x_{1}} v\right\|^{2} & \leq c \sum_{n=-\infty}^{+\infty} \frac{1}{n^{2}}\left[\left\|x_{0} \partial_{x_{1}} L_{n} c_{n}\right\|^{2}+\left\|i n \omega_{0} L_{n} c_{n}\right\|^{2}\right] \\
& \leq c\left[\left\|x_{0} \partial_{x_{1}} Q v\right\|^{2}+\left\|x_{0} \partial_{x_{2}} Q v\right\|^{2}\right]
\end{aligned}
$$

On the other hand, it results

$$
\begin{aligned}
0 & =\int_{\Omega_{T}^{\prime \prime}} \partial_{x_{1}} x_{1}\left(\partial_{x_{0}} v\right)^{2} d x \\
& =\int_{\Omega_{T}^{\prime \prime}}\left(\partial_{x_{0}} v\right)^{2} d x+\int_{\Omega_{T}^{\prime \prime}} 2 x_{1} \partial_{x_{0}} v \partial_{x_{0}} \partial_{x_{1}} v d x
\end{aligned}
$$

From which it follows

$$
\begin{aligned}
\left\|\partial_{x_{0}} v\right\|^{2} & =-2 \int_{\Omega_{T}^{\prime \prime}} x_{1}\left(\partial_{x_{0}} v\right)\left(\partial_{x_{0}} \partial_{x_{1}} v\right) d x \\
& \leq 2\left\|\partial_{x_{0}} v\right\|\left\|x_{1} \partial_{x_{0}} \partial_{x_{1}} v\right\| .
\end{aligned}
$$

Hence, we have

$$
\left\|\partial_{x_{0}} v\right\|^{2} \leq 4\left\|x_{1} \partial_{x_{0}} \partial_{x_{1}} v\right\|^{2}
$$

From (18) and (19), we deduce

$$
\left\|\partial_{x_{0}} v\right\|^{2} \leq c\left(\left\|x_{0} \partial_{x_{1}} Q v\right\|^{2}+\left\|x_{0} \partial_{x_{2}} Q v\right\|^{2}\right) .
$$

By using Lemma 1, it results

$$
\left\|\partial_{x_{0}} v\right\|^{2}+\|v\|^{2} \leq c\left(\left\|x_{0} \partial_{x_{1}} Q v\right\|^{2}+\left\|x_{0} \partial_{x_{2}} Q v\right\|^{2}\right) .
$$


Let us remark

$$
\begin{aligned}
& x_{0} \partial_{x_{1}} Q v=\sum_{n=-\infty}^{+\infty} x_{0} \partial_{x_{1}} L_{n} c_{n}\left(x_{0}, x_{1}\right) \frac{e^{i n \omega_{0} x_{2}}}{2 \sqrt{T}}, \\
& x_{0} \partial_{x_{2}} Q v=\sum_{n=-\infty}^{+\infty} i n \omega_{0} x_{0} L_{n} c_{n}\left(x_{0}, x_{1}\right) \frac{e^{i n \omega_{0} x_{2}}}{2 \sqrt{T}} .
\end{aligned}
$$

As a consequence, we have

$$
\begin{aligned}
& \left\|x_{0} \partial_{x_{1}} Q v\right\|_{]-2 T, 2 T[}^{2}=\sum_{n=-\infty}^{+\infty}\left|x_{0} \partial_{x_{1}} L_{n} c_{n}\right|^{2}, \\
& \left\|x_{0} \partial_{x_{2}} Q v\right\|_{]-2 T, 2 T[}^{2}=\sum_{n=-\infty}^{+\infty}\left|i n \omega_{0} x_{0} L_{n} c_{n}\right|^{2} .
\end{aligned}
$$

Furthermore, we obtain

$$
\begin{aligned}
& \left(x_{0} \partial_{x_{1}} Q v\right)\left(x_{2}\right)+\left(x_{0} \partial_{x_{1}} Q v\right)\left(-x_{2}\right)=\sum_{n=-\infty}^{+\infty} 2 x_{0} \partial_{x_{1}} L_{n} c_{n} \frac{\cos \left(n \omega_{0} x_{2}\right)}{2 \sqrt{T}} \\
& \left(x_{0} \partial_{x_{2}} Q v\right)\left(x_{2}\right)+\left(x_{0} \partial_{x_{2}} Q v\right)\left(-x_{2}\right)=\sum_{n=-\infty}^{+\infty} 2 i n \omega_{0} x_{0} L_{n} c_{n} \frac{\cos \left(n \omega_{0} x_{2}\right)}{2 \sqrt{T}} .
\end{aligned}
$$

By using (21), (22), (23) and (24), we deduce

$$
\begin{aligned}
&\left\|x_{0} \partial_{x_{1}} Q v\right\|_{]-2 T, 2 T[}^{2}+\left\|x_{0} \partial_{x_{2}} Q v\right\|_{]-2 T, 2 T[}^{2} \\
&=\sum_{n=-\infty}^{+\infty}\left|x_{0} \partial_{x_{1}} L_{n} c_{n}\right|^{2}+\sum_{n=-\infty}^{+\infty}\left|i n \omega_{0} x_{0} L_{n} c_{n}\right|^{2} \\
&=\left\|x_{0}\left(\partial_{x_{1}} Q v\right)\left(x_{2}\right)+x_{0}\left(\partial_{x_{1}} Q v\right)\left(-x_{2}\right)\right\|_{]-2 T, 2 T[}^{2} \\
& \quad+\left\|x_{0}\left(\partial_{x_{2}} Q v\right)\left(x_{2}\right)+x_{0}\left(\partial_{x_{2}} Q v\right)\left(-x_{2}\right)\right\|_{]-2 T, 2 T[}^{2} \\
&=2\left\|x_{0}\left(\partial_{x_{1}} Q v\right)\left(x_{2}\right)+x_{0}\left(\partial_{x_{1}} Q v\right)\left(-x_{2}\right)\right\|_{] 0,2 T[}^{2} \\
& \quad+2\left\|x_{0}\left(\partial_{x_{2}} Q v\right)\left(x_{2}\right)+x_{0}\left(\partial_{x_{2}} Q v\right)\left(-x_{2}\right)\right\|_{] 0,2 T[}^{2} \\
& \leq 2\left(\left\|x_{0}\left(\partial_{x_{1}} Q v\right)\left(x_{2}\right)\right\|_{] 0,2 T[}^{2}+\left\|x_{0}\left(\partial_{x_{1}} Q v\right)\left(-x_{2}\right)\right\|_{] 0,2 T[}^{2}\right. \\
&\left.+\left\|x_{0}\left(\partial_{x_{2}} Q v\right)\left(x_{2}\right)\right\|_{] 0,2 T[}^{2}+\left\|x_{0}\left(\partial_{x_{2}} Q v\right)\left(-x_{2}\right)\right\|_{] 0,2 T[}^{2}\right) .
\end{aligned}
$$

Now, we want to estimate directly the norms. Let us start from

$$
x_{0}\left(\partial_{x_{1}} Q v\right)\left(x_{2}\right)=\sum_{n=-\infty}^{+\infty} x_{0} \partial_{x_{1}} L_{n} c_{n}\left(x_{0}, x_{1}\right) \frac{e^{i n \omega_{0} x_{2}}}{2 \sqrt{T}}
$$


It results

$$
\begin{aligned}
& \left\|x_{0}\left(\partial_{x_{1}} Q v\right)\left(x_{2}\right)\right\|_{] 0,2 T[}^{2} \\
& =\frac{1}{4 T} \sum_{n=-\infty}^{+\infty} \sum_{h=-\infty}^{+\infty} x_{0}^{2} \partial_{x_{1}} L_{n} c_{n}\left(x_{0}, x_{1}\right)\left(\partial_{x_{1}} L_{h} c_{h}\left(x_{0}, x_{1}\right)\right)^{*} \int_{0}^{2 T} e^{i n \omega_{0} x_{2}} e^{-i h \omega_{0} x_{2}} d x_{2} .
\end{aligned}
$$

Let us compute the other norm remembering that

$$
x_{0}\left(\partial_{x_{1}} Q v\right)\left(-x_{2}\right)=\sum_{n=-\infty}^{+\infty} x_{0} \partial_{x_{1}} L_{n} c_{n}\left(x_{0}, x_{1}\right) \frac{e^{-i n \omega_{0} x_{2}}}{2 \sqrt{T}} .
$$

We have

$$
\begin{aligned}
& \left\|x_{0}\left(\partial_{x_{1}} Q v\right)\left(-x_{2}\right)\right\|_{] 0,2 T[}^{2} \\
& =\frac{1}{4 T} \sum_{n=-\infty}^{+\infty} \sum_{h=-\infty}^{+\infty} x_{0}^{2} \partial_{x_{1}} L_{n} c_{n}\left(x_{0}, x_{1}\right)\left(\partial_{x_{1}} L_{h} c_{h}\left(x_{0}, x_{1}\right)\right)^{*} \int_{0}^{2 T} e^{-i n \omega_{0} x_{2}} e^{i h \omega_{0} x_{2}} d x_{2} .
\end{aligned}
$$

From which, it follows

$$
\left\|x_{0}\left(\partial_{x_{1}} Q v\right)\left(x_{2}\right)\right\|_{] 0,2 T[}^{2}=\left\|x_{0}\left(\partial_{x_{1}} Q v\right)\left(-x_{2}\right)\right\|_{] 0,2 T[}^{2} .
$$

Moreover, making use of (25) and (20), we obtain

$$
\begin{aligned}
\left\|\partial_{x_{0}} v\right\|_{\Omega_{T}^{\prime \prime}}^{2}+\|v\|_{\Omega_{T}^{\prime \prime}}^{2} & \leq c\left(\left\|x_{0} \partial_{x_{1}} Q v\right\|_{\Omega_{T}^{\prime \prime}}^{2}+\left\|x_{0} \partial_{x_{2}} Q v\right\|_{\Omega_{T}^{\prime \prime}}^{2}\right) \\
& \leq 2 c\left(\left\|x_{0} \partial_{x_{1}} Q v\right\|_{\Omega_{T}^{\prime}}^{2}+\left\|x_{0} \partial_{x_{2}} Q v\right\|_{\Omega_{T}^{\prime}}^{2}\right)
\end{aligned}
$$

Since $v\left(x_{0}, x_{1}, x_{2}\right)=u\left(x_{0}, x_{1}, x_{2}-T\right)$, for every $\left(x_{0}, x_{1}, x_{2}\right) \in \Omega_{T}^{\prime}$, we have

$$
\left\|\partial_{x_{0}} u\right\|_{\Omega_{T}}+\|u\|_{\Omega_{T}} \leq c\left(\left\|x_{0} \partial_{x_{1}} Q u\right\|_{\Omega_{T}}+\left\|x_{0} \partial_{x_{2}} Q u\right\|_{\Omega_{T}}\right),
$$

from which the claim follows.

Let us remark that the positive constant $c$ in (26) does not depend on $T$ but only on $x_{1}$. As a consequence, the following result holds:

Corollary 1 For every $h, T>0$, there exists a positive constant $c$ such that

$$
\left\|\partial_{x_{0}} u\right\|+\|u\| \leq c\left(\left\|\partial_{x_{1}} Q u\right\|+\left\|\partial_{x_{2}} Q u\right\|\right)
$$

for every $u \in C_{0}^{\infty}(\bar{\Omega})$ such that $\operatorname{supp} u \subseteq[0, T[\times]-T, T[\times]-n T, n T[$, for every $n \in \mathbb{N}$. 


\section{A priori estimate in Sobolev spaces}

In the following, we establish a priori estimate in the Sobolev spaces.

Theorem 3 For every $s>0$, it results

$$
\left\|\partial_{x_{0}} u\right\|_{H^{0,0,-s}}+\|u\|_{H^{0,0,-s}} \leq c\left(\|Q u\|_{H^{0,1,-s}}+\|Q u\|_{H^{0,0,-s+1}}\right), \quad \forall u \in C_{0}^{\infty}\left(\Omega_{h, T}\right),
$$

where $\Omega_{h, T}=\left[0, h[\times]-T, T\left[^{2}\right.\right.$.

Proof Let $\varphi \in C_{0}^{\infty}(\mathbb{R})$ such that $\varphi(x)=1$ in $[-(n-1) T,(n-1) T]$ and $\operatorname{supp} \varphi \subseteq$ ] $-n T, n T$ [, with $n>1$. For every $u \in C_{0}^{\infty}\left(\Omega_{h, T}\right)$, we set $v_{s}=\varphi A_{s} u$, where $A_{s}$ is the pseudodifferential operator defined as:

$$
A_{s} u=\frac{1}{2 \pi} \int_{\mathbb{R}} e^{i x_{2} \xi_{2}}\left(1+\left|\xi_{2}\right|^{2}\right)^{-\frac{s}{2}} \widehat{u}\left(x_{0}, x_{1}, \xi_{2}\right) d \xi_{2},
$$

with $s>0$. Applying (27) to $v_{s}$, we have

$$
\begin{aligned}
\left\|\partial_{x_{0}} v_{s}\right\|+\left\|v_{s}\right\| \leq & c\left(\left\|\partial_{x_{1}} Q v_{s}\right\|+\left\|\partial_{x_{2}} Q v_{s}\right\|\right) \\
= & c\left(\left\|\partial_{x_{1}} Q \varphi A_{s} u\right\|+\left\|\partial_{x_{2}} Q \varphi A_{s} u\right\|\right) \\
\leq & c\left(\left\|\varphi \partial_{x_{1}} Q A_{s} u\right\|+\left\|\partial_{x_{1}}[Q, \varphi] A_{s} u\right\|\right) \\
& +c\left(\left\|\partial_{x_{2}} \varphi Q A_{s} u\right\|+\left\|\partial_{x_{2}}[Q, \varphi] A_{s} u\right\|\right) \\
\leq & c\left(\left\|\varphi \partial_{x_{1}} A_{s} Q u\right\|+\left\|\partial_{x_{1}}[Q, \varphi] A_{s} u\right\|\right) \\
& +c\left(\left\|\varphi \partial_{x_{2}} A_{s} Q u\right\|+\left\|\left[\partial_{x_{2}}, \varphi\right] Q A_{s} u\right\|+\left\|\partial_{x_{2}}[Q, \varphi] A_{s} u\right\|\right) \\
= & c\left(\left\|\varphi A_{s} \partial_{x_{1}} Q u\right\|+\left\|\varphi A_{s} \partial_{x_{2}} Q u\right\|+\left\|R_{1} Q u\right\|\right. \\
& \left.+\left\|R_{2} u\right\|+\left\|R_{3} \partial_{x_{0}} u\right\|\right)+c\left\|[Q, \varphi] A_{s} \partial_{x_{1}} u\right\|,
\end{aligned}
$$

where $R_{1}, R_{2}$ and $R_{3}$ are regularizing operators with respect to the variable $x_{2}$ of type

$$
R_{i}=\psi\left(\frac{x_{2}}{(n-1) T}\right) A_{s}, \quad i=1,2,3
$$

with $\psi \in C_{0}^{\infty}(\mathbb{R})$ such that $\psi=0$ in $[-1,1]$, as in Lemma 3, and having used $\partial_{x_{1}} Q A_{s} u=A_{s} \partial_{x_{1}} Q u$ and $\partial_{x_{2}} Q A_{s} u=A_{s} \partial_{x_{2}} Q u$.

Making use of Lemmas 1, 3 and (29), we deduce

$$
\begin{aligned}
\left\|\partial_{x_{0}} u\right\|_{H^{0,0,-s}}+\|u\|_{H^{0,0,-s}} \leq & c\left(\|Q u\|_{H^{0,1,-s}}+\|Q u\|_{H^{0,0,-s+1}}+\|Q u\|_{H^{0,0,-s}}\right) \\
& +c\left(\left\|R_{4} \partial_{x_{1}} u\right\|+\left\|R_{5} \partial_{x_{1}} \partial_{x_{0}} u\right\|\right),
\end{aligned}
$$

where $R_{4}$ and $R_{4}$ are regularizing operators with respect to the variable $x_{2}$ of type (30). 
Now, written the operator $Q$ as:

$$
Q u=L\left(\partial_{x_{0}} u\right)+x_{1}^{2} \partial_{x_{2}}^{2} \partial_{x_{0}} u+b x_{1}^{3} \partial_{x_{2}}^{3} u,
$$

where $L$ is the wave operator, namely $L=\partial_{x_{0}}^{2}+\partial_{x_{1}}^{2}$, it results

$$
\left(L\left(\partial_{x_{0}} u\right), \partial_{x_{0}}^{2} u\right)=\left(Q u, \partial_{x_{0}}^{2} u\right)-\left(x_{1}^{2} \partial_{x_{2}}^{2} \partial_{x_{0}} u, \partial_{x_{0}}^{2} u\right)-\left(b x_{1}^{3} \partial_{x_{2}}^{3} u, \partial_{x_{0}}^{2} u\right)
$$

Integrating by parts, we have easily:

$$
\left\|\partial_{x_{0}}^{2} u\right\|+\left\|\partial_{x_{1}} \partial_{x_{0}} u\right\| \leq c\left(\left\|\partial_{x_{2}}^{2} \partial_{x_{0}} u\right\|+\left\|\partial_{x_{2}}^{3} u\right\|+\|Q u\|\right) .
$$

Making use of Lemma 1, it follows

$$
\left\|\partial_{x_{0}} u\right\|+\left\|\partial_{x_{1}} u\right\|+\left\|\partial_{x_{0}}^{2} u\right\|+\left\|\partial_{x_{1}} \partial_{x_{0}} u\right\| \leq c\left(\left\|\partial_{x_{2}}^{2} \partial_{x_{0}} u\right\|+\left\|\partial_{x_{2}}^{3} u\right\|+\|Q u\|\right)
$$

Taking into account (31), (32) and Lemma 3, we deduce

$$
\begin{aligned}
\left\|\partial_{x_{0}} u\right\|_{H^{0,0,-s}}+\|u\|_{H^{0,0,-s}} \leq & c\left(\|Q u\|_{H^{0,1,-s}}+\|Q u\|_{H^{0,0,-s+1}}+\|Q u\|_{H^{0,0,-s}}\right) \\
& +c\left(\left\|\partial_{x_{1}} u\right\|_{H^{0,0,-s-3}}+\left\|\partial_{x_{1}} \partial_{x_{0}} u\right\|_{H^{0,0,-s-3}}\right) \\
\leq & c\left(\|Q u\|_{H^{0,1,-s}}+\|Q u\|_{H^{0,0,-s+1}}\right) \\
& +c\left(\|u\|_{H^{0,0,-s}}+\left\|\partial_{x_{0}} u\right\|_{H^{0,0,-s}}+\|Q u\|_{H^{0,0,-s}}\right) .
\end{aligned}
$$

From which we have

$$
\left\|\partial_{x_{0}} u\right\|_{H^{0,0,-s}}+\|u\|_{H^{0,0,-s}} \leq c\left(\|Q u\|_{H^{0,1,-s}}+\|Q u\|_{H^{0,0,-s+1}}\right)
$$

namely (28).

\section{Proof of Theorem 1}

For every $u \in C_{0}^{\infty}\left(\Omega_{h, T}\right)$, where $\Omega_{h, T}=\left[0, h[\times]-T, T\left[{ }^{2}\right.\right.$, let $\psi={ }^{t} Q u=Q u$ and let $F(\psi)=(f, u)$. It results

$$
|F(\psi)| \leq\|f\|_{H^{0,0, s}\left(\Omega_{h, T}\right)}\|u\|_{H^{0,0, s}\left(\Omega_{h, T}\right)} .
$$

Making use of (28), it follows

$$
\begin{aligned}
|F(\psi)| & \leq c\|f\|_{H^{0,0, s}\left(\Omega_{h, T}\right)}\left(\left\|{ }^{t} Q u\right\|_{H^{0,1,-s}\left(\Omega_{h, T}\right)}+\left\|{ }^{t} Q u\right\|_{H^{0,0,-s+1}\left(\Omega_{h, T}\right)}\right) \\
& \leq c^{\prime}\|\psi\|_{H^{0,1,-s+1}\left(\Omega_{h, T}\right)} .
\end{aligned}
$$


Hence, the functional $F$ can be extended in $H^{0,1,-s+1}\left(\Omega_{h, T}\right)$ and, therefore, there exists $w \in H^{0,-1, s-1}\left(\Omega_{h, T}\right)$ such that

$$
F(\psi)=(w, \psi)=\left(w,{ }^{t} Q u\right)=(g, u), \quad \forall u \in C_{0}^{\infty}\left(\Omega_{h, T}\right)
$$

Then, we have

$$
Q w=g, \quad \text { in } \mathcal{D}^{\prime}\left(\Omega_{h, T}\right) .
$$

Written $Q w=L\left(\partial_{x_{0}} w\right)+x_{1}^{2} \partial_{x_{2}}^{2} \partial_{x_{0}} w+b x_{1}^{3} \partial_{x_{2}}^{3} w$, we obtain

$$
L\left(\partial_{x_{0}} w\right)=g-x_{1}^{2} \partial_{x_{2}}^{2} \partial_{x_{0}} w-b x_{1}^{3} \partial_{x_{2}}^{3} w
$$

For $s>4$, we deduce $\partial_{x_{0}} w \in H^{0,0, s-1}$ and, hence, $u \in H^{1, s-1}$. Repeating the same procedure more times, we have that if $g \in H^{r}$ then $w \in H^{r-2}$. Therefore, if $r \geq 5$, we have

$$
\left(w,{ }^{t} Q u\right)=(g, u), \quad \forall u \in C_{0}^{\infty}\left(\Omega_{h, T}\right)
$$

Choosen a suitable $u$, for instance, such that $u\left(0, x^{\prime}\right)=0, \partial_{x_{0}} u\left(0, x^{\prime}\right)=0$ and $\partial_{x_{0}}^{2} u\left(0, x^{\prime}\right)=\varphi\left(x^{\prime}\right)$, with $\varphi \in C_{0}^{\infty}(]-T, T\left[^{2}\right)$, integrating by parts in the left-hand side of (33), we obtain

$$
(Q w, u)+\int_{[-T, T]^{2}} \varphi\left(x^{\prime}\right) w d x^{\prime}=(g, u) .
$$

As a consequence, we get

$$
\int_{[-T, T]^{2}} \varphi\left(x^{\prime}\right) w d x^{\prime}=0 .
$$

For the arbitrariness of $\varphi$, it follows

$$
w\left(0, x^{\prime}\right)=0
$$

Instead, choosing $u \in C_{0}^{\infty}\left(\Omega_{h, T}\right)$ such that $u\left(0, x^{\prime}\right)=0, \partial_{x_{0}} u\left(0, x^{\prime}\right)=\varphi\left(x^{\prime}\right)$ and $\partial_{x_{0}}^{2} u\left(0, x^{\prime}\right)=0$, with $\varphi \in C_{0}^{\infty}(]-T, T\left[^{2}\right)$, and proceeding as above, it results

$$
\partial_{x_{0}} w\left(0, x^{\prime}\right)=0
$$

Finally, if we chose $u \in C_{0}^{\infty}\left(\Omega_{h, T}\right)$ such that $u\left(0, x^{\prime}\right)=\varphi\left(x^{\prime}\right), \partial_{x_{0}} u\left(0, x^{\prime}\right)=0$ and $\partial_{x_{0}}^{2} u\left(0, x^{\prime}\right)=0$, with $\varphi \in C_{0}^{\infty}(]-T, T\left[^{2}\right)$, we obtain

$$
\partial_{x_{0}}^{2} w\left(0, x^{\prime}\right)=0
$$


Then we have proved that there exists $w \in H^{r-2}\left(\Omega_{h, T}\right)$, with $r \geq 5$, such that

$$
\left(w,{ }^{t} Q u\right)=(g, u), \quad \forall u \in C_{0}^{\infty}\left(\Omega_{h, T}\right)
$$

$w\left(0, x^{\prime}\right)=0, \partial_{x_{0}} w\left(0, x^{\prime}\right)=0$ and $\partial_{x_{0}}^{2} w\left(0, x^{\prime}\right)=0$. Hence, if $g \in H^{r}$, with $r \geq 5$, there exists a solution $w \in H^{r-2}$ to the problem

$$
\left\{\begin{array}{l}
Q w=g, \quad \text { in } \Omega_{h, T} \\
w\left(0, x^{\prime}\right)=0, \partial_{x_{0}} w\left(0, x^{\prime}\right)=0, \partial_{x_{0}}^{2} w\left(0, x^{\prime}\right)=0
\end{array}\right.
$$

where $g=i f$, with $f \in H_{l o c}^{r}(\Omega)$. Therefore there exists a solution to problem (1) also in $\Omega_{h, T}$.

\section{Conclusions}

The paper deals with a class of hyperbolic operators with triple characteristics. A priori estimate in Sobolev spaces with negative indexes are obtained. Thanks to this estimate, the existence of solutions to the associated Cauchy problem can be established.

Funding Open access funding provided by Università degli Studi di Napoli Federico II within the CRUICARE Agreement.

\section{Compliance with ethical standards}

Conflict of interest On behalf of all authors, the corresponding author states that there is no conflict of interest.

Open Access This article is licensed under a Creative Commons Attribution 4.0 International License, which permits use, sharing, adaptation, distribution and reproduction in any medium or format, as long as you give appropriate credit to the original author(s) and the source, provide a link to the Creative Commons licence, and indicate if changes were made. The images or other third party material in this article are included in the article's Creative Commons licence, unless indicated otherwise in a credit line to the material. If material is not included in the article's Creative Commons licence and your intended use is not permitted by statutory regulation or exceeds the permitted use, you will need to obtain permission directly from the copyright holder. To view a copy of this licence, visit http://creativecommons.org/licenses/by/4.0/.

\section{References}

1. Barbagallo, A., Esposito, V.: A global existence and uniqueness result for a class of hyperbolic operators. Ric. Mat. 63, 25-40 (2014)

2. Barbagallo, A., Esposito, V.: Energy estimates for the Cauchy problem associated to a class of hyperbolic operators with double characteristics in presence of transition. Ric. Mat. 64, 243-249 (2015)

3. Barbagallo, A., Esposito, V.: The Cauchy-Dirichlet problem for a class of hyperbolic operators with double characteristics in presence of transition. J. Math. Anal. Appl. 442, 149-170 (2016)

4. Barbagallo, A., Esposito, V.: On hyperbolic equations with double characteristics in the presence of transition. Bound. Value Probl. (2016). https://doi.org/10.1186/s13661-016-0646-z

5. Bernardi, E., Nishitani, T.: On the Cauchy problem for non-effectively hyperbolic operators, the Gevrey 5 well-posedness. J. Anal. Math. 105, 197-240 (2008) 
6. Bernardi, E., Nishitani, T.: Counterexamples to $C^{\infty}$ well posedness for some hyperbolic operators with triple characteristics. Proc. Jpn. Acad. 91(Ser. A), 19-24 (2015)

7. Kajitani, K., Wakabayashi, S., Nishitani, T.: The Cauchy problem for hyperbolic operators of strong type. Duke Math. J. 75, 353-408 (1994)

8. Nishitani, T.: Cauchy Problem for Noneffectively Hyperbolic Operators. MSJ Memoirs, vol. 30. Mathematical Society of Japan, Tokyo (2013)

9. Treves, F.: Introduction to Pseudodifferential and Fourier Integral Operators, vol. 1. Springer, Berlin (1980)

Publisher's Note Springer Nature remains neutral with regard to jurisdictional claims in published maps and institutional affiliations. 